\title{
Uma Abordagem Experimental para Avaliação da Melhoria de Processos
}

\author{
Reinaldo C. Silva Filho, Ana Regina C. Rocha, Guilherme H. Travassos \\ Programa de Engenharia de Sistemas e Computação - COPPE/UFRJ \\ Caixa Postal 68.511 - CEP 21945-970 - Rio de Janeiro - RJ - Brazil \\ \{cabral, darocha, ght\} @cos.ufrj.br
}

\begin{abstract}
This paper describes an experimentation based approach application regarding software process improvement. The obtained benefits had influenced the organization to demand new studies and improvements.

Resumo. Este artigo descreve a aplicação de uma abordagem baseada em experimentação à melhoria de processo de software. Os benefícios obtidos pela organização fomentaram a demanda por novos estudos e melhorias.
\end{abstract}

\section{Introdução}

Melhorar continuamente é uma questão de sobrevivência em um mundo globalizado e altamente competitivo. No âmbito organizacional o que se deseja é minimizar os custos, aumentar a produtividade e fornecer um produto com qualidade suficiente ${ }^{1}$ para diferenciar-se dos demais produtos concorrentes, qualquer que seja a área de atuação da organização.

Existem diversas abordagens para prover melhoria aos processos de software que, de uma forma geral, podem ser agrupadas em abordagens bottom-up e top-down [McGarry e Thomas 1994]. Em uma abordagem top-down a melhoria é considerada como uma mudança realizada nos processos para que se aproximem a um padrão genérico e amplamente aceito pelo grau de qualidade que pode prover à organização. Destacam-se aqui o modelo $\mathrm{CMMI}^{2}$, a série de normas ISO 9000, ISO/IEC 12207, ISO/IEC 15504 e o MR mps [Weber et al. 2004]. Em uma abordagem bottom-up a melhoria é obtida a partir da experiência e do conhecimento adquirido em projetos anteriores e de acordo com os produtos, objetivos e características da organização. As mudanças são realizadas considerando um escopo local e não práticas universalmente aceitas. Nesta categoria encontramos o Quality Improvement Paradigm (QIP) e Fábrica de Experiências [Basili 1995].

A escolha de uma dessas abordagens não é tarefa trivial. É possível, inclusive, combiná-las para constituir uma estratégia que considere as boas práticas dos modelos de qualidade e o contexto ao qual a organização está inserida. Rifkin (2001) sugere que o

\footnotetext{
${ }^{1}$ O termo "qualidade suficiente" refere-se à percepção de valor do produto que a organização possui mediante a possibilidade da adoção do produto para potencializar o seu negócio.

${ }^{2}$ Capability Maturity Model Integration, modelo proposto pelo SEI - Software Engineering Institute.
} 
sucesso da iniciativa de melhoria depende da estratégia de negócio adotada pela organização e o alinhamento da abordagem de melhoria a esta estratégia.

Esse trabalho apresenta uma abordagem baseada em experimentação para avaliar a melhoria de processo de software realizada por uma organização. A partir das questões consideradas prioritárias pela organização, que adotou uma estratégia bottom-up para empreender a melhoria dos processos de software, foram feitas alterações em determinado processo e estas foram avaliadas em um estudo que ilustrou os efeitos da mudança no processo e indicou novas oportunidades de melhorias.

Nas quatro seções subseqüentes é apresentada uma breve discussão sobre a experiência realizada e os resultados obtidos.

\section{A Maxma ${ }^{3}$ e sua estratégia de melhoria}

Considerando seu contexto e a diversidade de abordagens existentes em prol da melhoria, a Maxma elaborou uma estratégia adequada às suas necessidades e disponibilidade de recursos.

\subsection{Contexto da Organização}

Em 2001 a Maxma deparou-se com a necessidade de mensurar o tempo investido em cada atividade relacionada ao desenvolvimento de software. O objetivo era realizar uma caracterização das atividades e identificar oportunidades de melhoria.

A primeira iniciativa foi a criação de um mecanismo de controle para registro e acompanhamento do tempo investido nas atividades, o software GA - Gerenciador de Atividades. Além disso, processos de software inspirados na norma ISO/IEC 12207 e no CMMI foram estabelecidos com o objetivo de normatizar a execução das atividades e com o intuito de torná-las passíveis de comparação. Contudo não houve a preocupação de manter a conformidade com os modelos. O foco foi na construção de processos simples, fáceis de entender, executar, gerenciar e com um esforço dentro das possibilidades da organização, como sugerido em Dantekar (1997).

\subsection{A estratégia para melhoria adotada}

A partir de um levantamento periódico para identificar oportunidades de melhoria, a organização enumerou e identificou um conjunto de problemas (agrupados em áreas) que demandavam soluções. Uma lista de prioridades foi estabelecida com base em um conjunto de critérios (simplicidade da solução, custo, esforço, eficácia e prazo; com fatores de importância organizacional na faixa de 1-3) alinhados com os objetivos organizacionais. Equipes constituídas por profissionais experientes em cada área analisaram as causas mais prováveis e propuseram soluções para cada problema considerando os critérios estabelecidos.

O problema prioritário identificado: "Os prazos acordados com os clientes raramente são cumpridos".

\footnotetext{
${ }^{3}$ MAXMA - Cooperativa de Trabalho de Profissionais em Informática. Entidade de ciência e tecnologia autônoma, sem fins lucrativos, democraticamente gerida, tendo como área fim a atuação em TI.
} 
Durante a análise deste problema vários indícios foram coletados com o objetivo de identificar as principais causas: (i) Durante a execução do projeto algumas atividades importantes atrasam. Com isso outras atividades dependentes também atrasam. Ao final do projeto, somado todos os atrasos, é impossível cumprir o prazo previsto para a conclusão; (ii) Ausência de medidas corretivas para evitar o atraso de atividades importantes; (iii) Grande concentração de esforço em ações de contingência no final do projeto; (iv) Ausência de pontos de controle nos cronogramas.

A partir dos indícios, um conjunto de causas em potencial foi identificado. Em seguida, esse conjunto foi refinado sucessivas vezes e culminou com a eleição de uma das causas mais prováveis: “Deficiência do controle sobre o cronograma.”.

Após a eleição da causa mais provável, um conjunto de propostas de solução foi elaborado e, a partir deste, foram selecionadas as seguintes ações: (i) Inserir pontos de controle para indicar pontos críticos no cronograma; (ii) Alterar o Processo de Controle do Cronograma para interagir com o Processo de Resolução de Problemas do Projeto quando fossem observados atrasos nos pontos de controle.

Sendo assim, o Processo de Elaboração do Cronograma (PEC) e o Processo de Controle do Cronograma (PCC) passaram a contemplar duas novas atividades: (i) Identificar pontos críticos no cronograma para verificar a existência de atividades em atraso e (ii) Verificar atrasos e adiantamentos com relação aos pontos de controle definidos no cronograma.

Para avaliar os efeitos das alterações realizadas no PCC a organização optou pela realização de um estudo caracterizado por: (i) realizar uma avaliação a partir da primeira execução do novo processo, utilizando o projeto como piloto na observação dos efeitos da modificação; (ii) ser realizado no ambiente organizacional, com pouco controle sobre as variáveis do ambiente que poderiam influenciar o estudo; (iii) investigar uma simples entidade dentro de um espaço de tempo específico [Wohlin 2000]; (iv) analisar os dados tendo em vista a linha básica construída a partir do histórico de projetos da organização.

\section{3. $O$ estudo}

\subsection{Definição dos Objetivos}

Avaliar se a alteração no PCC foi eficaz no que tange a observância do prazo limite declarado no cronograma para conclusão dos projetos. A partir da execução de um projeto piloto, caracterizar o esforço dedicado à execução do projeto após a data limite estabelecida no cronograma.

\subsubsection{Objetivo do estudo}

Analisar o Processo de Controle do Cronograma.

Com o propósito de avaliar a alteração realizada no processo.

Com respeito à eficácia (prazo limite especificado no cronograma obedecido).

Do ponto de vista da organização.

No contexto da execução de projeto com o processo de software alterado. 


\subsubsection{Questão e Métricas}

Questão: Qual o esforço despendido a partir da data limite especificada no cronograma para a conclusão do projeto?

Métricas: (M1) Esforço total realizado: total de horas destinadas à execução de todas as atividades do projeto; (M2) Esforço investido no prazo: total de horas investidas até a data limite prevista para a conclusão do projeto; (M3) Total de horas destinadas à execução das atividades após a data prevista para conclusão do projeto.

\subsection{Planejamento}

\subsubsection{Definição das Hipóteses}

Sejam, Em - Percentual médio do esforço total despendido nos projetos após a data prevista para conclusão; e $\boldsymbol{E} \boldsymbol{e}$ - Percentual do esforço total do projeto despendido a partir da data limite especificada no cronograma para concluir o projeto.

Hipótese nula (H0): A alteração não influenciará a eficácia do processo. H0: $E m=E e$ Hipótese alternativa (H1): A alteração aumentará a eficácia do processo. H1: $E m>E e$ Hipótese alternativa (H2): A alteração diminuirá a eficácia do processo. H2: $E m<E e$

\subsubsection{Descrição da instrumentação}

A partir do software GA todos os dados relacionados ao cronograma puderam ser extraídos. Cada atividade do cronograma possuía um conjunto de atributos (data de início, data prevista para conclusão e esforço previsto em horas). As atividades eram associadas aos membros da equipe responsáveis por sua execução. Todas as tarefas realizadas em prol do cumprimento da atividade também foram registradas no GA (data, hora inicial, hora final, descrição da tarefa, e outros atributos). Durante o projeto o gerente executou a nova versão do PCC e, semanalmente, um relatório sobre a situação das atividades foi apresentado durante as reuniões de acompanhamento do projeto. Vale ressaltar que, nos pontos de controle, além do relato da situação das atividades, ações de contingência foram deflagradas, o que implicou no consumo de recursos não alocados previamente para o projeto.

\subsubsection{Seleção do Contexto}

O estudo foi caracterizado por um processo on-line, ocorrendo no ambiente de produção da organização. Os participantes foram profissionais do quadro da organização integrantes da equipe de desenvolvimento do projeto piloto. $\mathrm{O}$ estudo foi realizado em um problema real, representado por um produto feito sob encomenda. $\mathrm{O}$ estudo possui caráter específico devido às particularidades inerentes à organização, tais como cultura, comportamento, estrutura e outros aspectos.

\subsubsection{Seleção da População}

A seleção da população consistiu em aplicar o critério "o próximo projeto a ser desenvolvido", o que caracteriza a aleatoriedade da seleção. Vale ressaltar que, na ocasião, ainda não havia nenhuma previsão a respeito da dimensão, tipo ou qualquer outra característica do próximo projeto a ser contratado para desenvolvimento.

\subsubsection{Variáveis}

a) Variáveis independentes: Atributos do Processo de Controle de Processo: Responsável, Envolvidos, Ferramentas, Entradas, Saídas e Lista de Atividades. 


\section{Simpósio Brasileiro de Qualidade de Software}

b) Variáveis dependentes: Esforço total do projeto $(E t)$, total de horas investidas no projeto; Esforço investido ( $E i)$, total de horas investidas até a data limite; e Esforço excedente $(E e)$, percentual do esforço total despendido a partir da data limite especificada no cronograma para a conclusão do projeto, onde, $E e=100 *(1-(E i / E t))$.

\subsubsection{Validade}

a) Validade interna: A seleção do projeto piloto ocorreu ao acaso, utilizando como critério "o primeiro projeto contratado após a alteração do processo", desconsiderando qualquer característica, a exemplo de tamanho, prazo ou equipe alocada.

b) Validade de conclusão: A coleta das medidas foi realizada sem intervenção humana, a partir do software GA. Como estes registros eram auditados periodicamente para efeito da elaboração da folha de pagamento, o estudo assumiu que eram válidos.

c) Fatores de confusão: (i) A volatilidade dos requisitos: inicialmente imaginava-se que o retrabalho proporcionado pela volatilidade dos requisitos implicaria no incremento do esforço excedente $(E e)$. Contudo, de acordo com a definição do processo, verificou-se que esta volatilidade implicaria na reorganização do cronograma (redimensionamento das datas, inclusive a data prevista para conclusão); (ii) Imprecisão das estimativas: concluiu-se que sem o devido controle do cronograma do projeto, os atrasos poderiam continuar a acontecer, mesmo se as estimativas estivessem sendo realizadas com precisão. (iii) Formação dos participantes do projeto: a seleção dos membros da equipe executora do projeto ocorreu de forma indireta, através da seleção do projeto piloto. Com isso, verificou-se a inexistência da interferência do estudo no processo de escolha dos participantes, visto que a seleção do projeto também foi aleatória.

\subsection{Operação}

\subsubsection{Preparação e recursos utilizados}

Todos os integrantes da organização que assumem o papel de gerente dos projetos participaram da avaliação das propostas de melhoria para o processo. Isso dispensou a necessidade de treinamento ou orientação a respeito das alterações. Como o estudo não exerceu nenhuma influência sobre as atividades e o comportamento dos demais participantes da equipe, também não foi necessário prepará-los para a operação.

Foram utilizados os seguintes recursos no estudo: cliente MySQL, para conexão ao banco de dados do software GA; Software GA, para coleta de dados contidos nos relatórios de acompanhamento dos projetos; MS Excel, para apoiar a análise dos dados obtidos durante o estudo.

\subsubsection{Caracterização do projeto piloto}

- Identificação: VMX - Software de controle de inscrições de candidatos ao vestibular.

- Propósito: Fornecer uma interface web para interação com os usuários que vão se inscrever no processo seletivo para ingresso no ensino superior de uma instituição da rede privada. As facilidades incluem o cadastro, escolha do curso, emissão de bloquetos para pagamento da inscrição e acompanhamento do processo e divulgação dos resultados. $\mathrm{O}$ software também deve fornecer uma interface web para o usuário que monitora $o$ andamento das inscrições e duas outras para interação com o sistema de controle acadêmico (exportação dos candidatos aprovados) e com o sistema de correção de provas (exportação para gerar cartões-resposta e importação dos resultados). 


\section{Simpósio Brasileiro de Qualidade de Software}

- Cronograma: Início para 15/03/2004 e conclusão prevista para 14/06/2004. Pontos de controle inseridos nas datas de 19/05 e 02/06.

- Esforço: Previsão de 231 horas. Porém, o esforço investido até a data prevista para conclusão foi de 272 horas e o esforço total realizado 328h 29 min.

- Equipe: 6 membros graduados em Ciência da Computação, sendo 2 especialistas em Tecnologia da Informação.

- Gerência com experiência em 4 projetos de porte similar e com formação de especialista em Tecnologia da Informação.

- Processo de Desenvolvimento Maxma Versão 1.1 e linguagem Java.

- Demanda: externa.

\subsubsection{Construção da linha básica}

A linha básica dos projetos da organização não contemplava o percentual médio do esforço despendido nos projetos após a data prevista para conclusão $(E m)$. Quanto maior o valor de Em maior o esforço excedente dos projetos a partir da data prevista para sua conclusão. Seu cálculo pode ser expresso por:

$\frac{\sum_{i=1}^{\mathrm{n}}\left(100 \times\left(1-\left(\frac{E i_{i}}{E t_{i}}\right)\right)\right)}{n}$ , onde, $n$ é o número de projetos da amostra; $E t_{i}$, o esforço total do projeto $i$ em horas; e $E i_{i}$ esforço total em horas investidas na execução do projeto $i$ até a data limite prevista no cronograma.

A extração da métrica foi feita a partir do histórico dos projetos desenvolvidos na organização. O critérios foram: (i) projeto ter sido concluído até o início do estudo; e (ii) completude dos dados no GA para a extração das métricas. Foram agrupados 13 projetos de acordo com o processo de desenvolvimento utilizado.

Tabela 1. Caracterização dos projetos da base de dados do GA

\begin{tabular}{|c|c|c|c|c|c|}
\hline Grupo & Projetos & Processo & Tipo & Demanda & Linguagem \\
\hline I & A, B, C, D, E & 1.0 & web & Externa & Java \\
\hline II & F, G & 1.1 & web & Externa & Java \\
\hline III & H, I, Q, R, S, & Ad hoc & web $(\mathrm{H}, \mathrm{T})$ & Externa (I,Q,R,S $)$ & Java (H), VB(I), \\
& T & & $\begin{array}{c}\text { convencional } \\
(\mathrm{I}, \mathrm{Q}, \mathrm{R}, \mathrm{S})\end{array}$ & Interna $(\mathrm{H}, \mathrm{T})$ & $\begin{array}{c}\text { Delphi }(\mathrm{Q}, \mathrm{R}, \mathrm{S}), \\
\text { PHP }(\mathrm{T})\end{array}$ \\
\hline
\end{tabular}

Tabela 2. Percentual do esforço médio excedido por grupo (Em)

\begin{tabular}{|c|c|c|c|c|c|c|}
\hline Grupo & Id & $\begin{array}{l}\text { Tamanho } \\
\text { da Equipe }\end{array}$ & $\begin{array}{c}\text { Esforço } \\
\text { total } \\
\text { (horas) }\end{array}$ & $\begin{array}{c}\text { Esforço além da } \\
\text { data prevista } \\
\text { (horas) }\end{array}$ & $\begin{array}{c}\text { Percentual } \\
\text { excedido }\end{array}$ & $\begin{array}{l}\text { Esforço } \\
\text { médio } \\
\text { excedido }\end{array}$ \\
\hline \multirow{5}{*}{ I } & $\mathrm{A}$ & 9 & 1773 & 922 & $52 \%$ & \multirow{5}{*}{$\begin{array}{c}27,6 \% \\
(\mathrm{DP}=21,29 \%)\end{array}$} \\
\hline & B & 9 & 1224 & 269 & $22 \%$ & \\
\hline & $\mathrm{C}$ & 9 & 425 & 204 & $48 \%$ & \\
\hline & $\mathrm{D}$ & 9 & 492 & 34 & $7 \%$ & \\
\hline & $\mathrm{E}$ & 9 & 493 & 44 & $9 \%$ & \\
\hline \multirow[t]{2}{*}{ II } & $\mathrm{F}$ & 7 & 739 & 81 & $11 \%$ & \multirow{2}{*}{$\begin{array}{c}23 \% \\
(\mathrm{DP}=16,97 \%)\end{array}$} \\
\hline & $\mathrm{G}$ & 8 & 626 & 219 & $35 \%$ & \\
\hline \multirow{6}{*}{ III } & $\mathrm{H}$ & 1 & 267 & 45 & $17 \%$ & \multirow{6}{*}{$\begin{array}{c}25,17 \% \\
(\mathrm{DP}=16,15 \%)\end{array}$} \\
\hline & I & 3 & 275 & 39 & $14 \%$ & \\
\hline & $Q$ & 2 & 585 & 199 & $34 \%$ & \\
\hline & $\mathrm{R}$ & 2 & 174 & 9 & $5 \%$ & \\
\hline & $\mathrm{S}$ & 2 & 74 & 19 & $26 \%$ & \\
\hline & $\mathrm{T}$ & 1 & 71 & 39 & $55 \%$ & \\
\hline
\end{tabular}

DP: Desvio Padrão 
Para compor a linha básica a ser utilizada na verificação das hipóteses utilizaremos o valor obtido por $E m_{I I}$ (Tabela 3), dada a similaridade das características do projeto piloto com o segundo grupo da amostra. Portanto, $E m=E m_{I I}$.

\subsubsection{Execução}

A operação do estudo consistiu na extração das métricas a partir do banco de dados do software GA e da interação com o próprio software. Nenhuma modificação no software foi realizada. Todos os atributos necessários à execução do estudo já haviam sido contemplados no software. Através da execução de consultas SQL (ANSI) ao banco de dados os dados foram extraídos para arquivos de texto e organizados em planilhas.

\subsection{Análise e interpretação dos dados}

\subsubsection{Cálculo do Ee no projeto piloto}

Os procedimentos adotados para extração dos dados para calcular Ee foram os mesmos adotados para extração dos dados durante a construção da linha básica.

$$
E e=100 *(1-(272 / 328,48)), \text { ou seja, } E e=17,19 \%
$$

\subsubsection{Verificação das hipóteses}

Hipótese nula (H0): Em = Ee. Como H0: $23 \neq 17,19$. Portanto, H0 foi recusada. Hipótese alternativa (H1): Em > Ee. Como H1: $23>$ 17,19. Portanto, H1 foi aceita. Hipótese alternativa (H2): Em < Ee. Como H2: $23>17,19$. Portanto, H2 foi recusada.

\subsubsection{Análise Qualitativa}

Ao realizar a análise qualitativa sobre os dados coletados foi observado que: (i) Parte das horas que excederam o projeto $(37 \mathrm{~h} 7 \mathrm{~min})$ foi provocada por uma solicitação do cliente, que postergou algumas atividades para além do prazo previsto para conclusão do projeto. Desconsiderando estas horas no cômputo de $E e$, temos, $E e=100 *(1-1$ $272 / 291,36))=100 *(1-0,93355)=\mathbf{6 , 6 4} \%$. Este valor ainda menor para $E$ e reforça a refutação das hipóteses $\mathbf{H 0}$ e $\mathbf{H 2}$, sendo inclusive menor que o valor mínimo utilizado para construção da linha básica; (ii) $\mathrm{O}$ esforço realizado até a data limite prevista para conclusão do projeto $(272 \mathrm{~h}$ ) foi superior ao estimado (231h). Ou seja, houve uma concentração de horas anterior à data limite para conclusão do projeto piloto. Isto pode indicar que a precisão das estimativas de tempo e esforço precise ser melhorada.

\subsection{Empacotamento e apresentação dos resultados}

Nesta etapa do estudo, todas os dados e informações geradas em todas as etapas do estudo, foram devidamente organizadas para permitir o reuso e servir de referência em outros estudos e constituir a base de ativos da organização. Em seguida, houve uma apresentação dos resultados, de forma que a organização pudesse visualizar os resultados e outras oportunidades de melhoria em decorrência das análises realizadas.

\section{O esforço para realização do estudo}

O esforço dos desenvolvedores foi o que possuiu impacto direto nos custos do projeto, já que o tempo utilizado para a coleta concorre com o tempo destinado à realização das tarefas do projeto. Esta iniciativa onerou o projeto em torno de 3,6\% do custo total. 


\section{Conclusão}

A experimentação é uma poderosa ferramenta que pode ser utilizada em prol da avaliação da melhoria de processos na indústria. Além dos resultados relacionados à avaliação da mudança do processo, a análise qualitativa realizada forneceu indícios que sugerem a necessidade de aumentar a precisão das estimativas de tempo e esforço para os projetos. Considerando os dados computados no estudo, provavelmente, caso o processo não tivesse sido alterado, o esforço investido após a data limite prevista para conclusão do projeto seria acrescido de no mínimo 12\%, o que poderia trazer (ou ampliar) os transtornos para o cliente.

A execução deste estudo permitiu observar que: (i) ter acesso irrestrito aos dados de projeto é imprescindível; (ii) possuir mecanismos para coleta automatizada das medidas é fundamental; e (iii) a execução do estudo de forma adequada e imparcial provê auto-conhecimento à organização e fomento a novos estudos.

Com o intuito de nortear outros estudos utilizando a abordagem relatada neste trabalho, sugerimos o roteiro composto por seis passos: (i) entender o problema, (ii) definir os objetivos da avaliação, (iii) planejar o estudo, (iv) operar o estudo conforme o planejado, (v) analisar e interpretar os dados e (vi) empacotar o estudo e apresentar os resultados. Finalizando, ressaltamos que, além das dificuldades inerentes a realização do estudo em um ambiente de produção, óbices relacionados à cultura organizacional e confidencialidade dos dados foram extremamente visíveis durante sua execução.

\section{Agradecimentos}

À Maxma por autorizar a publicação dos dados e resultados do estudo realizado.

\section{Referências}

Basili, V. R., Caldiera, G., "Improve Software Quality by Reusing Knowledge and Experience", Sloan Management Review, Fall 1995, Vol. 37, N. 1, p. 55-64.

Dantekar, A., Perry, D. E., Votta, L. G., "Studies in Process Simplification", Software Process: Improvement and Practice, Vol 3, N. 2, p. 87-104, Junho, 1997.

McGarry, F. E., Thomas, M., "Top-Down vs. Bottom-Up Process Improvement", IEEE Software, p. 12-13, Julho, 1994.

Rifkin, S., "Why Software Process Innovations Are Not Adopted", IEEE Software, p. 110-112, Julho/Agosto, 2001.

Weber, K. C., Rocha, A.R., Alves, A., Ayala, A. M. A., Gonçalves, A., Paret, B., Salviano, C., Machado, C. F., Petit, D., Araújo, E., Barroso, M. G., Oliveira, K., Oliveira, L. C. A., Amaral, M. P., Campelo, R. E. C., Maciel, T., "Modelo de Referência para Melhoria de Processo de Software: uma abordagem brasileira", XXX Conferência Latino-Americana de Informática - CLEI2004, Peru, Setembro, 2004.

Wohlin, C., "Experimentation in Software Enginneering - An introduction", Kluwer Academic Publishers, Massachusetts, USA, 2000. 